\title{
The Blooming of Natural Medicine
}

\section{Rafeek HI*}

Consultant in Alternative Medicine, Naturopathic Iridologist \& Specialist in Pain Management, Sri Lanka

*Corresponding author: Hameed Mohamed Rafeek, Consultant in Alternative Medicine, Naturopathic Iridologist \& Specialist in Pain Management, Advisor to state ministry of health and indigenous medicine, Sri Lanka, Tel: 0094 -779544505; Email: dr_hm_rafeek@hotmail.com

\section{Editorial}

Volume 3 Issue 1

Received Date: March 26, 2019

Published Date: March 29, 2019

DOI: $10.23880 /$ jonam-16000172

\section{Abstract}

There are two systems of health care available in this world-Western medicine and Alternative Medicine. But for patients looking for better health, there are a number of therapies / medicines wherever available such as Allopathy, Homeopathy, Osteopathy, Naturopathy-but whatever the patient needs sympathy/empathy. Looking on to these points, today conventional medicine is superb when it comes to surgery, emergency and trauma but there is no question that Alternative Medicine works better for just about everything else specially for chronic disease.

Keywords: Allopathy; Homeopathy; Osteopathy; Alternative Medicine

\section{Editorial}

When it comes to natural medicine, people prefer it since it natural value in it and has no side effects compared to chemical medicine. What I want to highlight is that if one continues taking the chemical medicines, it will affect the organs and diminish the lifespan gradually. We must drink more water and it should be alkaline water and also the diet should include more fruits, vegetables and organic food, which will increase your lifespan.

My research findings, treatment in some cases is more cost effective over long term because it emphasizes prevention and goes after causes rather than symptoms. Many alternative method works by assisting your body by healing yourself instead of strong drugs.

One characteristic of orthodox medicine is that there is no clear relationship between the patients subjective experience of ill health or health and objective experience as measured scientifically, For example: a person may have raised blood pressure and may not know because he doesn't feel unwell .on the other hand, the patient with irritable bowel syndrome may know very well that he or she is extremely unwell, yet there is nothing to measure. So there are always differences between the subjective and objectives experience of ill health. Another characteristic of orthodox medicine worth mentioning is that elements of the environment are not often taken into account. For example, until recently Orthodox doctors in the UK have little or no training whatsoever in the value of nutrition. However this is changing largely due to input of other professions than medicine and input from psychology, occupational therapy, physiotherapy, speech therapy, naturopathic iridologist and other professions, which are supporting the health care service in the National Health Service (NHS) of UK and other Europe countries. So, in the orthodox medicine greater emphasis is now being placed on the role of the family and other relationships at work on the individual's health. The impact of quality of housing, whether the person is employed and the nature of his employment, nutrition and diet are all important internal factors, such as the role 


\section{Journal of Natural \& Ayurvedic Medicine}

of emotions in our health, of stress and the importance of self-expression, attitude and beliefs are beginning to be as important.

It is important to classify and to stratify CAM. There are the longstanding ethnic medical systems, the Chinese medicine, which includes Acupuncture, Herbal medicine, Physical Medicine, Magnetic therapy ,Color therapy Cupping Therapy and other modalities which have very long established histories 3000/4000 years old, perhaps still developing of course, medicine and is extensively used. The other domain, Ethnic Medicine, that is the traditional Indian, Sri Lankan or Ayurveda medicine, again has a very long well established history and in its own country, especially and Osteopathy and Chiropractic, one often asks, what is the difference between these two? The reality is that the difference is now much less than it was and both therapies work with the muscles, ligaments, soft tissue and the purpose is to align and reshape the body to give it good use. The mind body therapist, the Hypnotherapy healing technique, Radionics, the Creative therapy, by which one means Art, Music, Sound, Yoga, Drama therapy are extensively used in the national health services in the UK. Anthroposophysical medicine is a form of medicine, which is extensively used by Europe but not elsewhere. Examples of these are, naturopathy, and European tradition of using the patient own resources through diet, meditation and exercise, to come back to good health then. There are other two systems:
- Herbal medicine which is on a worldwide basis is the most challenges and pharmaceutical approach of medicines widely.

- Homeopathy is that form of therapy which most challenges the pharmaceutical approach of medicine in that the principals are: like should treat like and that of the diluted medicine, to the extent those immeasurable parts of the original medicines are more powerful than the original medicine.

Under the banner of complementary system, diagnosing stools are pulse diagnosis, iris diagnosis, tongue diagnosis, facial diagnosis, Su-Jok diagnosis, Auricular diagnosis. According to the meridian electronic technique devices leads to correct differentiation organs deficiency, or energy deficiency or excess can be identified by stools or determine information about bodily constitutional strength or weakness.

We concluded there is a trend in the whole world and the world is coming back to natural remedies or integrated medicines a way of treatment whether the west or east the world is coming back to natural life , when the time needful pharmaceutical drugs need to be taken. Today the world is growing up with alternative and complementary medicine due to vast benefits for life long, supporting the scientific researches related to alternative and complementary medicine training to western doctors to learn the benefits of treatment and practices to understand about complementary medicine. 